\title{
Pulmonary Mycobacterium kansasii infection: comparison of the clinical features, treatment and outcome with pulmonary tuberculosis
}

\author{
S A Evans, A Colville, A J Evans, A J Crisp, I D A Johnston
}

\begin{abstract}
Background - In the United Kingdom Mycobacterium kansasii is the most common pulmonary non-tuberculous mycobacteria to cause disease in the non-HIV positive population.

Methods - The clinical features, treatment, and outcome of 47 patients (13 women) of mean (SD) age 58 (17) years with culture positive pulmonary $M$ kansasii infection were compared with those of 87 patients ( 23 women) of mean (SD) age 57 (16) years with culture positive pulmonary $M$ tuberculosis infection by review of their clinical and laboratory records. Each patient with $M$ kansasii infection was matched for age, sex, race and, where possible, year of diagnosis with two patients with $M$ tuberculosis infection.

Results - All those with M kansasii infection were of white race. Haemoptysis was more common in patients infected with $M$ kansasii but they were less likely to present as a result of an incidental chest radiograph or symptoms other than those due to mycobacterial infection. Patients with M kansasii were also less likely to have a history of diabetes, but the frequency of previous chest disease and tuberculosis was similar. An alcohol intake of $>14$ units/week was less frequent in those with $M$ kansasii, but there were no significant differences in drug history, past and present smoking habit, occupational exposures, social class, or marital status. Patients with $M$ kansasii received a longer total course of antimycobacterial therapy and, in particular, extended treatment with ethambutol and rifampicin was given. There was no significant difference in outcome between pulmonary $M$ kansasii or $M$ tuberculosis infection.
\end{abstract}

Conclusions - There are group differences between the clinical features of the two infections but, with the possible exception of diabetes and alcohol intake, these features are unlikely to be diagnostically helpful. Treatment of M kansasii infection with ethambutol, isoniazid, and rifampicin in these patients was as effective as standard regimens given to patients infected with M tuberculosis.

(Thorax 1996;51:1248-1252)

Keywords: Mycobacterium kansasii, Mycobacterium tuberculosis, pulmonary infection, clinical features, treatment.
Mycobacterium kansasii is the most common cause of pulmonary non-tuberculous mycobacterial infection in the non-HIV population of the United Kingdom with 40-65 isolations (pulmonary and non-pulmonary) annually. ${ }^{1}$ Isolation rates for $M$ kansasii appear to be stable in the UK, perhaps reflecting its environmental source. $M$ kansasii has frequently been isolated from tap water and is thought to be acquired from the environment rather than from case to case transmission. $^{2-6}$

Unlike Mycobacterium tuberculosis, $M$ kansasii both infects and colonises the respiratory tract. Criteria for pulmonary infection include isolation (preferably multiple) with clinical and radiological findings compatible with mycobacterial disease. ${ }^{78}$ Infection with $M$ kansasii has therapeutic implications as the organism shows in vitro resistance to pyrazinamide and often isoniazid. Drug regimens that include rifampicin and ethambutol are recommended ${ }^{89}$ and, when treated appropriately, outcome is good. ${ }^{10-13}$ However, the recent British Thoracic Society trial of treatment for nine months with rifampicin and ethambutol alone reported a relapse rate of $9 \%$ over a follow up period of five years. ${ }^{13}$

It has been suggested previously that there are differences in the presentation of pulmonary infection with non-tuberculous mycobacteria and $M$ tuberculosis ${ }^{814}$ but no systematic study has been published. Such differences, if present, would not only be of scientific interest, but would also be of potential clinical value. ${ }^{15}$ Clinical suspicion of $M$ kansasii infection might allow ethambutol and rifampicin to be included in the initial drug regimen while awaiting culture results. Differentiating the two infections on clinical grounds might also have implications for contact tracing.

We have therefore performed a comparison of the clinical features, treatment, and outcome of pulmonary $M$ kansasii and $M$ tuberculosis infections diagnosed in Nottingham.

\section{Methods}

All mycobacterial culture for the Nottingham area is undertaken in the Nottingham Public Health Laboratory using standard methods. ${ }^{16}$ Sputum smears stained with auramine are examined by fluorescent microscopy and the presence of acid fast organisms confirmed with Ziehl-Neelsen stain. Lowenstein-Jensen slopes are incubated for 12 weeks. All mycobacterial isolates are referred to the Public Health Laboratory Service Mycobacterial Reference Unit at Cardiff for identification and sensitivity testing. 
Table 1 Symptoms at presentation in patients infected with Mycobacterium kansasii and Mycobacterium tuberculosis

\begin{tabular}{lll}
\hline & $\begin{array}{l}\text { Mycobacterium } \\
\text { kansasii } \\
(n=47)\end{array}$ & $\begin{array}{l}\text { Mycobacterium } \\
\text { tuberculosis } \\
(n=87)\end{array}$ \\
\hline Chest pain & $16(34)$ & $25(29)$ \\
Breathlessness & $24(51)$ & $52(60)$ \\
Cough & $43(91)$ & $78(90)$ \\
Sputum & $40(85)$ & $74(85)$ \\
Haemoptysis & $15(32)^{*}$ & $11(13)^{*}$ \\
Weight loss & $25(53)$ & $54(62)$ \\
Sweats/fevers & $8(17)$ & $20(23)$ \\
\hline
\end{tabular}

Values in parentheses are percentages. ${ }^{*} \mathrm{p}<0.02$.

All respiratory isolations of $M$ kansasii between 1979 and 1990 were identified from laboratory records $(n=50)$. Case notes were available for 49 patients. Any patient with isolations of $M$ kansasii but no radiographic evidence of mycobacterial disease was excluded from the study $(n=1)$, as were any patients known to be HIV positive $(n=1)$. The 1980 Office of Population Census and Surveys classification of occupations was used to define social class. ${ }^{17}$

Patients from whom respiratory isolates of $M$ tuberculosis were obtained over the same period were identified from laboratory records. Each patient infected with $M$ kansasii was matched with two patients with $M$ tuberculosis infection for age, sex, race and, if possible, year of diagnosis. Of 100 patients identified by these means, records were available for 87 . Race was initially taken from the family name and subsequently reviewed on receipt of the case notes. A questionnaire on clinical features, treatment, and outcome was completed from the case notes by a single investigator, additional information being obtained from laboratory records.

The EpiInfo surveillance package was used for statistical analysis. ${ }^{18}$ The clinical features of the two groups were compared using $\chi^{2}$ tests (with Yates' correction where appropriate) and Fisher's exact probability test. The level of statistical significance was taken as $<0.05$.

\section{Results}

The mean age of the patients with $M$ kansasii infection was 58 (range 26-87) years and 13 of the 47 were women. The mean age of those infected with $M$ tuberculosis was 57 (range 2688 ) years with 23 of the 87 being women. All patients with isolates of $M$ kansasii were white and therefore were matched with white patients infected with $M$ tuberculosis.

The presenting symptoms of the two groups showed no significant differences, with the exception of haemoptysis which was more common in those with pulmonary $M$ kansasii infection (table 1). $M$ kansasii was less likely to present as a result of an incidental chest radiograph ( 0 versus $7, p<0.05)$ or because of symptoms other than those of mycobacterial infection ( 1 versus $11, \mathrm{p}<0.05$ ).

Marital status, past and present smoking, and social class were not significantly different between the two groups, though data collection for social class was incomplete due to poor
Table 2 Past and present medical problems in the two groups of patients

\begin{tabular}{|c|c|c|}
\hline & $\begin{array}{l}\text { Mycobacterium } \\
\text { kansasii } \\
(n=47)\end{array}$ & $\begin{array}{l}\text { Mycobacterium } \\
\text { tuberculosis } \\
(n=87)\end{array}$ \\
\hline Any lung disease & $27(57)$ & $38(44)$ \\
\hline Previous tuberculosis & $9(19)$ & $15(17)$ \\
\hline Asthma & $5(11)^{*}$ & $1(1)^{*}$ \\
\hline COPD & $9(19)$ & $15(17)$ \\
\hline Diabetes & $0(0)^{*}$ & $9(10)^{*}$ \\
\hline Malignancy & $4(9)$ & $6(7)$ \\
\hline Cardiac disease & $14(30)$ & $19(22)$ \\
\hline GI peptic & $8(17)$ & $19(22)$ \\
\hline GI other & $6(13)$ & $5(6)$ \\
\hline \multirow{3}{*}{$\begin{array}{l}\text { Past alcohol > } 14 \text { units/week } \\
\text { Present alcohol }>14 \text { units/ } \\
\text { week }\end{array}$} & $4(9)^{*}$ & $31(36)^{*}$ \\
\hline & & \\
\hline & $0(0)^{*}$ & $27(31)^{*}$ \\
\hline
\end{tabular}

Values in parentheses are percentages.

$\mathrm{COPD}=$ chronic obstructive pulmonary disease; GI peptic $=$ $\mathrm{COPD}=$ chronic obstructive pulmonary disease; GI peptic $=$
peptic ulceration, gastritis, oesophagitis or reflux; GI other $=$ peptic ulceration, gastritis, oesophagitis or reflux; GI
gastrointestinal disease other than GI peptic. ${ }^{*} p<0.05$.

documentation in the case notes (data available for 39 in the $M$ kansasii group and 70 in the $M$ tuberculosis group).

A comparison of past and concurrent medical problems is shown in table 2. Patients with $M$ kansasii were less often diabetic (0 versus 9 , $\mathrm{p}<0.05)$ but were more likely to have asthma ( 5 versus $1, \mathrm{p}<0.05$ ). A history of alcohol intake of $>14$ units/week was found more commonly in the $M$ tuberculosis group (31 versus 4, $\mathrm{p}<0.05)$. Otherwise, history of other diseases including previous tuberculosis was not significantly different between the groups, nor were there significant differences in drug history. In particular, past and previous use of immunosuppressive agents including steroids was similar.

Laboratory investigations including erythrocyte sedimentation rate, haemoglobin, and white cell count were similar in the two groups, as was the frequency of abnormal liver function tests.

Patients infected with $M$ kansasii had a median of three sputum samples examined (range $1-9)$ and 29 (63\%) had at least one smear positive result. Patients with $M$ tuberculosis infection also had a median of three sputum samples examined (range 1-8) with 56 (64\%) having at least one smear positive result. All $M$ kansasii isolates were sensitive to rifampicin and ethambutol (one borderline sensitivity). Twelve were sensitive, 31 were of borderline sensitivity, and four were resistant to streptomycin. All were resistant to isoniazid and pyrazinamide resistance was assumed. All $M$ tuberculosis isolates were sensitive to rifampicin, isoniazid, ethambutol, pyrazinamide (only 58 tested), and streptomycin (one borderline sensitivity).

There was no significant difference in the daily dosage of any antimycobacterial agent used in the treatment of $M$ kansasii or $M$ tuberculosis. The total duration of antimycobacterial chemotherapy was significantly longer for $M$ kansasii (mean 10.3 months (range 0-22)) than for $M$ tuberculosis (8.5 months (range $0-24) ; \mathrm{p}=0.001$ ). The most striking difference between the two groups was the prolonged use of ethambutol in the treatment of $M$ kansasii (table 3 ). No second line antimycobacterial drugs were used. 
Table 3 Treatment duration in the two groups

\begin{tabular}{|c|c|c|c|c|c|c|c|}
\hline \multirow[b]{2}{*}{ Drug } & \multicolumn{3}{|c|}{ Mycobacterium kansasii } & \multicolumn{3}{|c|}{ Mycobacterium tuberculosis } & \multirow[b]{2}{*}{$p$ value } \\
\hline & $\begin{array}{l}\text { Number of } \\
\text { patients }\end{array}$ & $\begin{array}{l}\text { Mean duration } \\
\text { (months) }\end{array}$ & Range & $\begin{array}{l}\text { Number of } \\
\text { patients }\end{array}$ & $\begin{array}{l}\text { Mean duration } \\
\text { (months) }\end{array}$ & Range & \\
\hline Rifampicin & 39 & 10.3 & $0-22$ & 73 & 8.4 & $0-24$ & 0.001 \\
\hline Isoniazid & 34 & 7.1 & $0-22$ & 73 & 8.5 & $0-24$ & NS \\
\hline Ethambutol & 39 & 9.2 & $0-18$ & 51 & 2.1 & $0-6$ & $<0.001$ \\
\hline Pyrazinamide & 21 & 1.5 & $0-4$ & 43 & 2.2 & $0-9$ & 0.001 \\
\hline
\end{tabular}

Table 4 Causes of death

\begin{tabular}{lll}
\hline & $\begin{array}{l}\text { Mycobacterium } \\
\text { kansasii } \\
(n=47)\end{array}$ & $\begin{array}{l}\text { Mycobacterium } \\
\text { tuberculosis } \\
(n=87)\end{array}$ \\
\hline Mycobacterial infection & 3 & 8 \\
Malignant disease & 4 & 3 \\
Vascular disease & 2 & 3 \\
Gastrointestinal bleed & 1 & 2 \\
Miscellaneous/unknown & 1 & $4^{*}$ \\
\hline *One road traffic accident. & &
\end{tabular}

The follow up period for patients who survived the first month after treatment was started was 5-108 months (mean 37) for $M$ kansasii infected patients and 2-104 months (mean 25.5) for patients infected with $M$ tuberculosis. No relapses were detected. Overall there was no significant difference in the survival of the two groups (10 with $M$ kansasii died compared with 20 with $M$ tuberculosis) nor in the mean (SD) age at diagnosis of those who died ( $M$ kansasii 72.2 (9.1) years versus $M$ tuberculosis 67.5 (16.8) years; $\mathrm{p}=0.7$ ).

Of the 10 patients who died in the $M$ kansasii group, four died before antimycobacterial therapy was given. In two of these, one with Hodgkin's lymphoma and one with thyrotoxicosis, the mycobacterial infection was thought to have significantly contributed to death. In the other two cases death was ascribed to carcinoma of the lung and heart failure. Three patients died during treatment after three weeks, one month, and 11 months of treatment. In two cases the cause of death was carcinoma of the lung, but in the patient who died after three weeks of treatment the cause of death given on the death certificate was tuberculosis. Three patients died after finishing treatment, none being attributed to mycobacterial disease.

Of the 20 patients who died in the $M$ tuberculosis group, death occurred in 13 patients before or during the first month of antituberculous treatment. In seven of these the cause of death given on the death certificate was tuberculosis. There were seven late deaths but in only one (a patient who refused to comply with treatment) was $M$ tuberculosis cited as the cause. The causes of death are given in table 4 .

\section{Discussion}

An ability to differentiate between pulmonary infections caused by $M$ kansasii or $M$ tuberculosis on clinical grounds would have important therapeutic implications, particularly the early introduction of ethambutol for suspected $M$ kansasii infection. ${ }^{15}$ This would be particularly important in the presence of rifampicin resistance, but this appears to be rare at present. ${ }^{13}$
While several authors have referred to clinical differences between pulmonary $M$ kansasii and $M$ tuberculosis, ${ }^{8914}$ the present study represents the first direct comparison of such features. We have shown that the clinical features of pulmonary $M$ kansasii infection are generally similar to pulmonary $M$ tuberculosis, but that differences do exist. Diabetes and heavy alcohol consumption appear to be rare in patients with $M$ kansasii. The presence of these features, however, merely reinforces the already high prior likelihood of $M$ tuberculosis, given that $M$ tuberculosis was isolated eight times more frequently than $M$ kansasii in the white population of Nottingham during the study period. Conversely, though haemoptysis and asthma were more common in the $M$ kansasii group, the differences are not sufficiently great to have an impact on making a positive diagnosis of $M$ kansasii. The relative isolation rates mentioned above mean that any particular patient with haemoptysis is still more likely to have $M$ tuberculosis, and a patient with asthma has a similar risk of being infected with $M$ tuberculosis or $M$ kansasii. We have shown that it is not possible to differentiate reliably between pulmonary $M$ kansasii infection and $M$ tuberculosis infection on radiological grounds. ${ }^{19}$ We now conclude that it is also not possible to predict the presence of pulmonary $M$ kansasii infection on clinical grounds and that the group differences observed are unlikely to be clinically relevant.

All 47 isolates of $M$ kansasii in our series came from patients of white race, most of whom were men $(72 \%)$. The effect of race is difficult to interpret as only $8 \%$ of the population of Nottingham are from ethnic minorities, but the data suggest that $M$ kansasii may be less common in the non-white population. Studies in the USA have also noted the predominance of white men in pulmonary $M$ kansasii infection. ${ }^{8}$ The reasons for this are unclear.

Haemoptysis has previously been noted to occur in $20-30 \%$ of patients with $M$ kansasii infection $^{1012}$ and the present study has confirmed haemoptysis as a common symptom $(31 \%)$, occurring more frequently than in pulmonary $M$ tuberculosis infection. Possible reasons for this include the degree of endobronchial disease and the erosion of bronchial vessels by cavitation. While there are no data on the relative incidence of endobronchial disease, the incidence of cavitation is probably similar. ${ }^{19}$ However, others have reported cavitation to be more common in pulmonary $M$ kansasii infection than other pulmonary mycobacterial infections. ${ }^{2021}$ Mycetomas, a potential confounding cause of haemoptysis, are probably less common in patients infected with $M$ kan- 
sasii than with $M$ tuberculosis and are uncommon on presentation. ${ }^{192122}$ An official statement from the American Thoracic Society states that in lung disease caused by non-tuberculous mycobacteria fever and weight loss are less common and less severe than in $M$ tuberculosis. ${ }^{8}$ This unreferenced statement is at variance with the findings of the present study which shows weight loss and fever to be as common in $M$ kansasii infection as in $M$ tuberculosis.

Previous reports have often emphasised the high rate of pre-existing lung disease and, in particular, past tuberculosis in pulmonary $M$ kansasii infection. ${ }^{914}$ Again, these previous studies were not controlled comparisons, and we have found no significant difference in the incidence of these features between $M$ kansasii and $M$ tuberculosis infections.

By contrast, diabetes and a past or present heavy alcohol intake were unequivocally commoner in pulmonary $M$ tuberculosis than pulmonary $M$ kansasii infection. Associations between $M$ tuberculosis and both diabetes and alcoholism have been noted previously, ${ }^{23-25}$ which perhaps is not surprising given that impaired host responses have been observed in both conditions. ${ }^{26-28}$ Why diabetes and alcoholism are less common in pulmonary $M$ kansasii infection than $M$ tuberculosis is unclear, however. It is possible that immunological changes influence susceptibility to reactivation to a greater degree than susceptibility to new mycobacterial disease.

Microbiologically it is possible to give an early indication of $M$ kansasii infection. Because of the morphological features of $M$ kansasii isolates ( $M$ kansasii is a photochromagen which turns orange on exposure to light), experienced laboratories can suggest that an isolate may be $M$ kansasii soon after growth is seen without waiting for reference laboratory reports. Even earlier guidance may be offered if characteristically beaded acid fast organisms are seen on the sputum smear. ${ }^{29}$ However, such advice is only possible if a laboratory isolates sufficient mycobacteria to maintain expertise and should otherwise be interpreted with caution.

The most striking difference in the management of the two groups is the longer period of antimycobacterial drug treatment given to patients with $M$ kansasii, and particularly the prolonged use of ethambutol. Banks et al ${ }^{10}$ suggested that 9-15 months of treatment with rifampicin and ethambutol was effective for $M$ kansasii infection. Our data suggest that the isolation of $M$ kansasii resulted in a change of treatment plan to conform with this finding.

The outcome over the period of follow up in this study was not significantly different for $M$ tuberculosis and $M$ kansasii. All deaths caused by mycobacterial disease occurred either before or soon after treatment was started. These data provide support for the use of prolonged treatment regimens including rifampicin, isoniazid, and ethambutol in the treatment of $M$ kansasii. The regimens used in the treatment of $M$ tuberculosis are well researched and offer a useful benchmark for comparison. The number of deaths occurring during chemotherapy (three of $43,7 \%$ ) was similar to the $5 \%$ observed during the British Thoracic Society (BTS) trial..$^{13}$ The BTS study of the treatment of pulmonary $M$ kansasii observed a relapse rate of $9 \%$ after a regimen of nine months of rifampicin and ethambutol. ${ }^{13}$ This contrasts with the relapse rate of zero for $M$ kansasii in the present study. A possible reason for this discrepancy is that our relapse rate is calculated on less complete follow up and retrospective data. Another possibility, however, is that more prolonged treatment, particularly of isoniazid (seven months in the present study compared with 2-4 months in the BTS study) led to a lower relapse rate. The fact that all $M$ kansasii isolates displayed in vitro resistance to isoniazid does not discount the role of isoniazid as synergy between antimycobacterial drugs is well documented ${ }^{30}$ and discrepancy between in vitro sensitivity and treatment response is also recognised. ${ }^{31}$

We conclude that differences between the clinical presentation of pulmonary $M$ kansasii and $M$ tuberculosis infections do exist but are unlikely to be clinically useful in the early identification of patients with pulmonary $M$ kansasii infection. Our data support the prolonged use of ethambutol, rifampicin, and isoniazid for treatment of $M$ kansasii infections. The choice of this regimen at the earliest opportunity relies on good liaison with the laboratory.

1 Campbell IA, Jenkins PA. Opportunist mycobacterial infections. In: Brewis RAL, Gibson GJ, Geddes DM, eds. Respiratory medicine. London: Balliere Tindall, 1990:1027342 .

2 McSwiggan DA, Collins $\mathrm{CH}$. The isolation of $M$ kansasii and M xenopi from water systems. Tubercle $1974 ; 55: 291-7$. Bailey RK, Wyles S, Dingley M, Hesse F, Kent GW. The isolation of high catalase Mycobacterium kansasii from tap water. Am Rev Respir Dis 1970;101:430-1.

4 Steadham JE. High catalase strains of Mycobacterium kansasii isolated from water in Texas. $\mathcal{F}$ Clin Microbiol 1980;11: 496-8.

5 Pang SC. Mycobacterium kansasii infections in Western Australia (1962-1987). Respir Med 1991;85:213-8.

6 Schraufnagel DE, Leech JA, Pollak B. Mycobacterium kansasii; colonization and disease. Br $\mathcal{F}$ Dis Chest 1986;80: 131-7.

7 Jenkins PA. Nontuberculous mycobacteria and disease. Eur 7 Respir Dis 1981;62:69-71

8 Diagnosis and treatment of disease caused by nontuberculous mycobacteria: official statement of the Amer-
tumosis and treatment ican Thoracic Society. Am Rev Respir Dis 1990;142: 940-53.

9 Ellis ME. Mycobacteria other than Mycobacterium tuberculosis. Curr Opin Infect Dis 1988;1:252-71.

10 Banks J, Hunter AM, Campbell IA, Jenkins PA, Smith AP. Pulmonary infections with Mycobacterium kansasii in Wales, 1970-9: review of treatment and response. Thorax 1983;38:271-4.

11 Rauscher CR, Kerby G, Ruth WE. A ten-year clinical experience with Mycobacterium kansasii. Chest 1974;66: 17-19.

12 Clague HW, El-Ansary EH, Hopkins CA, Roberts C. Pulmonary infection with opportunist mycobacteria on Merseyside 1974-83. Postgrad Med $\mathcal{f}$ 1986;62:363-8.

13 British Thoracic Society, Research Committee. Mycobacterium kansasii pulmonary infection: a prospective study of the results of nine months of treatment with rifampicin and ethambutol. Thorax 1994;49:435-6.

14 Wolinsky E. Nontuberculous mycobacteria and associated diseases. Am Rev Respir Dis 1979;119:107-59.

5 Davies PDO. Infection with Mycobacterium kansasii. Thorax 1994;49:435-6.

16 Collins CH, Grange JM, Yates MD. Organization and practice in tuberculous bacteriology. London: Butterworths, 1985. OPCS. Classification of occupations 1980. London: HMSO, 1980 .

18 Dean AD, Dean JA, Burton JH, Dicker RC. Epi Info, version 5: $A$ word processing, data base, and statistics programme 5: A word processing, data base, and statistics programme

19 Evans AJ, Crisp AJ, Hubbard RB, Colville A, Evans SA, Johnston IDA. Pulmonary Mycobacterium kansasii infoction: comparison of the radiological appearances with pulmonary tuberculosis. Thorax 1996;51:1243-1247.
pulion: comparison of the radiological appearances with 
20 O'Brien RJ, Geiter LJ, Snider DE. The epidemiology of nontuberculous mycobacterial diseases in the United States. Am Rev Respir Dis 1987;135:1007-14.

21 Christensen EE, Dietz GW, Ahn CH, Chapman JS, Murry RC, Hurst GA. Radiographic manifestations of pulmonary Mycobacterium kansasii infections. AfR 1978;131:985-93.

22 Maliwan N, Zvetina JR. Pulmonary mycetoma following Mycobacterium kansasii. infection. Arch Intern Med 1985; $145 \cdot 2180-3$

23 Chaves AD, Robins AB, Abeles H. Tuberculosis: case finding among homeless men in New York City. Am Rev Respir Dis 1961;84:900-1.

24 Rhodes RJ, Hames GH, Cambell MD. The problem of alcoholism among hospitalized tuberculosis patients. $\mathrm{Am}$ Rev Respir Dis 1969;99:440-2.

25 Hendy M, Stableforth D. The effect of established diabetes mellitus on the presence of infiltrative pulmonary tuberculosis in the immigrant asian community of an inner city area of the United Kingdom. Br $\mathcal{F}$ Dis Chest 1983;77: 87-93.

26 MacGregor RR. Alcohol and immune defense. $7 \mathrm{Am} \mathrm{Med}$ Assoc 1986;256:1474-9.

27 Faustman D, Eisenbarth G, Daley J, Breitmeyer J. Abnormal T-lymphocyte subsets in type 1 diabetes. Diabotes 1989 38:1462-8.

28 Buschard K, Ropke C, Madsbad S, Mehlsen J, Sorensen $T$, Rygaard J. Alterations in T lymphocyte subpopulation in patients with insulin dependent (type 1) diabetes mellitus. F Clin Lab Immunol 1983;10:127-31.

29 Grange JM. Mycobacterial and human disease. London: Edward Arnold, 1988: 34-5.

30 Banks J, Jenkins PA. Combined versus single antituberculosis drugs on the in vitro sensitivity patterns of nontuberculous mycobacteria. Thorax 1987;42:838-42.

31 Banks J, Jenkins PA, Smith AP. Pulmonary infection with Mycobacterium malmoense - a review of treatment and response. Thorax 1985;40:197-203. 